Revta brasil. Bot., São Paulo, V.24, n.1, p.1-9, mar. 2001

\title{
Variação na estrutura e na composição de Bromeliaceae em cinco zonas de restinga no Parque Nacional da Restinga de Jurubatiba, Macaé, RJ
}

\section{LUCIANA COGLIATTI-CARVALHO ${ }^{1,2}$, ANDRÉ FELIPPE NUNES DE FREITAS ${ }^{1,2}$, CARLOS FREDERICO DUARTE DA ROCHA ${ }^{1,3}$ e MONIQUE VAN SLUYS ${ }^{1}$}

(recebido: 31 de janeiro de 2000; aceito: 26 de setembro de 2000)

\begin{abstract}
Variation in structure and composition of Bromeliaceae at five zones of "restinga" in Jurubatiba National Park, Macaé, RJ). We studied the bromeliads at the Jurubatiba National Park in five vegetation zones: psamophilous halophilous creeper vegetation (PHR); closed rear beach (FPP); open shrubby Clusia (AAC); open shrubby Ericaceae (AAE) and temporarily flooded forest (MPI). We sampled 98 plots of $100 \mathrm{~m}^{2}(10 \times 10 \mathrm{~m})$ recording the bromeliad species and abundance, density, mass, richness, species diversity, evenness, and similarity among the zones. We estimated the amount of water reserved inside the bromeliads in each zone and by bromeliad species. We found a total of ten bromeliad species at the Jurubatiba National Park that occurred differently along the vegetation zones, with a total density of 10386 ind.ha $^{-1}$. No bromeliads were found in the PHR zone. The FPP zone had the highest total mass $\left(7721.2 \mathrm{~kg}^{-h^{-1}}\right)$ and Bromelia antiacantha was the most abundant, having the highest mass $\left(1366.5 \mathrm{~kg}^{-h^{-1}}\right)$. The AAE zone had the highest total bromeliad density $\left(15725\right.$ ind.ha $\left.^{-1}\right)$, the highest bromeliad diversity $\left(\mathrm{H}^{\prime}=2.358\right)$, evenness $(0.786)$ and amount of water reserved inside bromeliads (3294.8 L.ha ${ }^{-1}$ ). In the MPI zone, Aechmea bromeliifolia was the most abundant, being found exclusively in this zone. The highest similarity occurred between zones AAC and AAE (88.9\%). We conclude that the structure and composition of the bromeliads at the Jurubatiba National Park varied consistently among the vegetation zones of the restinga, and each zone is characterised by a different set of dominant bromeliads and different abundance distribution.
\end{abstract}

RESUMO - (Variação na estrutura e na composição de Bromeliaceae em cinco zonas de restinga no Parque Nacional da Restinga de Jurubatiba, Macaé, RJ). Estudamos as bromeliáceas do Parque Nacional da Restinga de Jurubatiba, Macaé, RJ, nas cinco diferentes zonas de vegetação: psamófila halófila reptante (PHR), fechada pós-praia (FPP), arbustiva aberta de Clusia (AAC), arbustiva aberta de ericácea (AAE) e mata periodicamente inundada (MPI). Analisamos abundância, densidade, biomassa, riqueza, diversidade, equitabilidade e similaridade de espécies entre as zonas. Estimamos a quantidade de água reservada no interior das bromélias por zona de restinga e por espécie de bromélia. Em 98 parcelas de $100 \mathrm{~m}^{2}(10 \mathrm{X} 10 \mathrm{~m})$ cada, registramos as espécies de bromélias, a abundância de cada espécie e a zona amostrada. A quantidade de água armazenada e a biomassa das bromélias foram estimadas medindo-se o volume de água reservada no vaso e pesando 10 indivíduos por espécie. Encontramos 10 espécies na restinga de Jurubatiba, com densidade total estimada de 10386 ind.ha ${ }^{-1}$. Na zona PHR, não ocorreu nenhuma espécie de bromélia. Na FPP, ocorreu a maior biomassa total $\left(7721,2 \mathrm{~kg}^{-h^{-1}}\right)$, sendo Bromelia antiacantha a mais abundante e com maior biomassa $\left(1366,5 \mathrm{~kg} \cdot \mathrm{ha}^{-1}\right)$. Na AAE, ocorreu a maior densidade total $\left(15725\right.$ ind.ha $\left.^{-1}\right)$, diversidade $\left(H^{\prime}=2,358\right)$, equitabilidade $(0,786)$ e quantidade de água reservada no interior de bromélias $\left(3294,8 \mathrm{~L} . h a^{-1}\right)$. Na MPI, Aechmea bromeliifolia foi a mais abundante, sendo exclusiva desta zona. A maior similaridade ocorreu entre AAC e AAE $(88,9 \%)$. Concluímos que a estrutura e a composição de Bromeliaceae da restinga de Jurubatiba variam fortemente entre as zonas estudadas, com cada zona possuindo um conjunto particular de espécies com diferentes distribuições de abundância.

Key words - Bromeliad species diversity, abundances distribution, Jurubatiba National Park, restinga

\section{Introdução}

As restingas recobrem cerca de $79 \%$ da costa brasileira (Lacerda et al. 1993), onde se estendem desde estreitas até extensas faixas de areia, como no litoral norte do Estado do Rio de Janeiro (Martins et al. 1993). O bioma de restinga possui uma vegetação

1. Universidade do Estado do Rio de Janeiro, IBRAG, Setor de Ecologia, Rua São Francisco Xavier, 524, 20550-011 Rio de Janeiro, RJ.

2. Universidade Federal do Rio de Janeiro, Programa de pósgraduação em Ecologia.

3. Autor para correspondência: cfdrocha@uerj.br característica devido a uma combinação de fatores físicos e químicos destas regiões, tais como elevada temperatura, salinidade, grande deposição de salsugem e alta exposição à luminosidade (Ormond 1960, Franco et al. 1984, Henriques et al. 1984). Nas imediações de fontes de água, como lagoas e braços das mesmas, a vegetação torna-se mais densa, formando florestas (Araujo et al. 1998).

As comunidades vegetais das restingas da costa brasileira são conhecidas quanto a sua composição florística e às formações vegetais nelas encontradas (e.g. Pfadenhauer 1978, Araujo \& Henriques 1984, Silva \& Somner 1984, Henriques et al. 1986, Araujo \& Oliveira 1988, Pereira \& Araujo 1995, Araujo et al. 1998), havendo, contudo, poucos trabalhos sobre 
a ecologia das espécies vegetais (Ormond 1960, Henriques et al. 1984, Waechter 1985, Zaluar 1997). Estas comunidades vegetais podem ser subdivididas em diferentes zonas, caracterizadas pela topografia, fisionomia e florística local específica de cada zona (Dansereau 1947, Ormond 1960). Alguns estudos têm sido realizados com comunidades vegetais em restingas, buscando compreender o padrão de zonação das espécies (e.g. Henriques et al. 1984) e diferentes fatores tais como a salsugem, a salinidade do solo e o movimento da areia têm sido sugeridos como responsáveis pela zonação das espécies vegetais (e.g. Ormond 1960, Franco et al. 1984, Henriques et al. 1986).

O Parque Nacional da Restinga de Jurubatiba (PNRJ), no litoral norte do Estado do Rio de Janeiro, ocupa uma extensão de cerca de $60 \mathrm{~km}$ ao longo da costa (Araujo et al. 1998). Diversos estudos têm sido realizados na área do PNRJ (e.g. Montezuma 1997, Zaluar 1997, Araujo et al. 1998), sendo o mesmo caracterizado fisionômica e floristicamente em dez zonas.

A família Bromeliaceae é muito representada em restingas (Silva \& Somner 1984, Henriques et al. 1986, Fontoura et al. 1991, Araujo 1992), sendo importante para a comunidade como um todo, principalmente pela capacidade de armazenar água em seu vaso, o que a torna um elemento importante para a manutenção da diversidade deste habitat (Picado 1913, Lopez 1997, Rocha et al. 1997). Diversas espécies animais utilizam as bromélias para forrageamento, reprodução e refúgio contra predadores (Smart 1938, Brito-Pereira et al. 1988, Rivero 1989, Oliveira et al. 1994a, b, Oliveira \& Rocha 1997). A germinação e o desenvolvimento de algumas espécies de plantas podem, também, ocorrer nas bromélias (Fialho 1990, Fialho \& Furtado 1993).

Estudou-se a comunidade de bromeliáceas em uma área da restinga de Jurubatiba, analisando a distribuição de abundâncias, a densidade, a biomassa, a riqueza e a diversidade da área como um todo e de cada uma das zonas, estimando, também, a equitabilidade e a similaridade na composição de espécies entre as diferentes zonas vegetais desta restinga. Adicionalmente, para avaliar a importância das bromélias como reservatórios de água livre nesta restinga, estimou-se o volume total de água reser- vado no interior das bromélias em cada zona de restinga e para cada espécie de bromélia.

\section{Material e métodos}

Área de estudo - O estudo foi realizado em março de 1999 e janeiro de 2000 (estações chuvosas), em uma área da restinga próxima à Lagoa de Cabiúnas, no Parque Nacional da Restinga de Jurubatiba, ( $22^{\circ} 17^{\prime} \mathrm{S}$ e $\left.41^{\circ} 41^{\prime} \mathrm{W}\right)$, que abrange os município de Macaé, Carapebus e Quissamã, litoral norte do Estado do Rio de Janeiro (figura 1). Esta região é dominada por uma lagoa de água doce (Lagoa Feia) e por uma planície arenosa (Araujo et al. 1998). A área de estudo é caracterizada por uma acentuada sazonalidade no regime de chuvas, com precipitação mensal mínima de aproximadamente $40 \mathrm{~mm}$, ocorrendo no inverno, e máxima de aproximadamente $190 \mathrm{~mm}$ no verão, com uma deficiência de água no solo entre os meses de junho e setembro. A temperatura média anual é de $22,6^{\circ} \mathrm{C}$, com a máxima atingindo $29,7^{\circ} \mathrm{C}$ em janeiro e, a mínima, $20,0^{\circ} \mathrm{C}$ em julho (Henriques et al. 1986, Araujo et al. 1998).

Uma primeira classificação desta restinga foi feita por Henriques et al. (1984), sendo posteriormente modificada e acrescida por Araujo et al. (1998). No presente trabalho, estudaram-se as populações de bromélias em cinco zonas propostas por Araujo et al. (1998): vegetação psamófila e halófila reptante (PHR), que se inicia próxima à escarpa praial, em faixa com largura de aproximadamente 5-10 m; arbustiva fechada de pós-praia (FPP), que é vegetação densa e lenhosa de difícil penetração; arbustiva aberta de Clusia (AAC), que se limita com a anterior e é composta por moitas densas de variados tamanhos; arbustiva aberta de Ericaceae (AAE), que apresenta moitas de vários tamanhos e irregulares, mais ou menos alinhadas em faixas; e mata periodicamente inundada (MPI), que sofre inundação periódica do solo, com dominância de Tabebuia cassinoides. Na área de estudo, estas zonas de vegetação são paralelas à linha de praia e o distanciamento do mar segue a sequiência em que foram apresentadas (figura 2).

A partir da zona PHR, foram realizadas sete transecções perpendiculares ao mar em direção ao interior da restinga, até atingir o fim da zona MPI, que estabelecia o fim da transecção; cada transecção estava separada de outra paralela por $50 \mathrm{~m}$. Nas

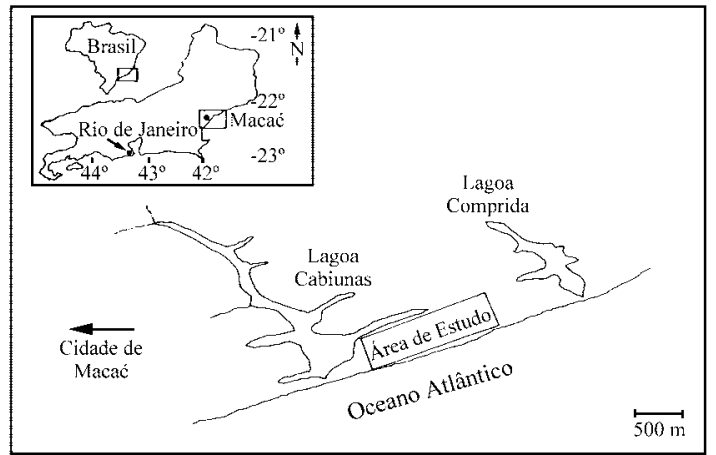

Figura 1. Localização da área de estudo no Parque Nacional da Restinga de Jurubatiba, Macaé, RJ. Modificado de Petrucio \& Faria (1998). 


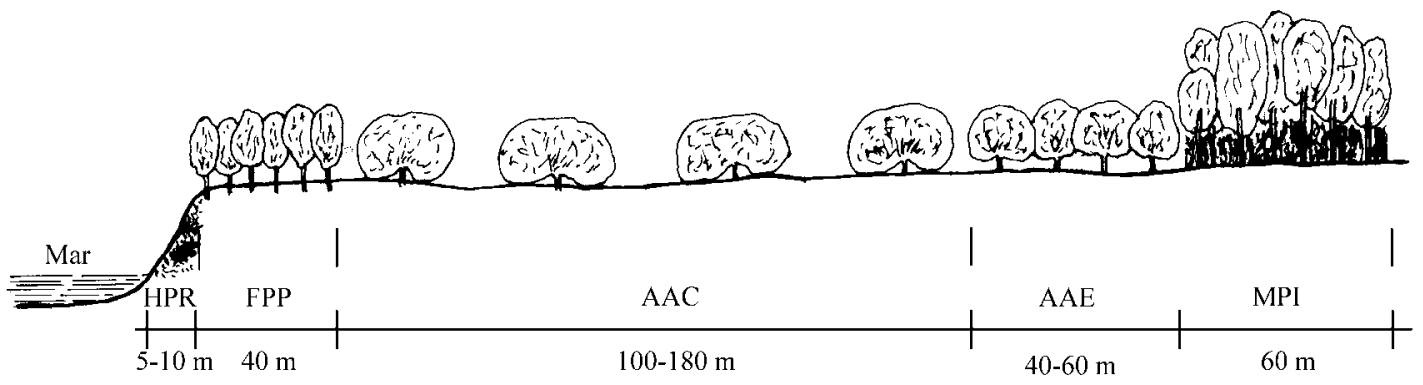

Figura 2. Perfil das zonas de vegetação estudadas no Parque Nacional da Restinga de Jurubatiba. HPR = halófila e psamófila reptante; FPP = fechada de pós-praia; AAC = área aberta de Clusia $; \mathrm{AAE}=$ área aberta de Ericácea; $\mathrm{MPI}$ = mata periodicamente inundada.

transecções, foram demarcadas parcelas de $100 \mathrm{~m}^{2}(10 \times 10 \mathrm{~m})$ distantes entre si $10 \mathrm{~m}$. Foram estabelecidas 98 parcelas, totalizando 0,98 ha de área amostrada. As parcelas foram distribuídas nas cinco zonas de vegetação da restinga descritas acima: sete na PHR (total de 0,07 ha), 14 na FPP (total de 0,14 ha), 44 na AAC (total de 0,44 ha), 16 na AAE (total de 0,16 ha) e 17 na MPI (total de 0,17 ha). Em cada parcela, foram registradas as espécies de bromélias presentes e o número de indivíduos de cada uma das espécies encontradas. Para estimar a densidade $\left(\right.$ ind.ha $\left.^{-1}\right)$ de bromélias por zona, dividiu-se o número total de bromélias amostradas nas parcelas de cada zona pela área total amostrada em cada zona (em ha). A diferença entre as densidades de bromélias em cada zona de restinga foi testada através do teste qui-quadrado de aderência (Magurran 1988). Para cada espécie, calculou-se a abundância relativa dividindo-se o número de indivíduos da espécie pela abundância total de bromélias amostradas, e a freqüência de ocorrência foi calculada através da divisão do número de parcelas em que a espécie ocorreu pelo número total de parcelas.

Calculou-se a diversidade através do índice de Shannon (H') e a equitabilidade (J) em cada zona analisada (Magurran 1988). A similaridade na composição entre as cinco zonas foi estimada através do índice de similaridade de Jaccard (Magurran 1988).

Para estimar o volume médio de água armazenada no vaso de cada espécie de bromélia, utilizaram-se dez indivíduos de cada uma das espécies, atentando para que fossem amostrados indivíduos de diferentes tamanhos e que estivessem em locais variados na restinga (sombreados ou mais expostos). A fim de minimizar os efeitos da sazonalidade (estação seca/chuvosa), o estudo foi realizado na época de chuvas. O volume de água no interior de cada bromélia foi obtido invertendo-se a bromélia em um saco plástico de 100 litros até que encerrasse qualquer gotejamento. $\mathrm{O}$ volume de água encontrado foi medido com provetas de 1 litro (com precisão de $10 \mathrm{~mL}$ ) e de $500 \mathrm{~mL}$ (com precisão de $5 \mathrm{~mL}$ ). Estimou-se a quantidade de água armazenada por cada espécie de bromélia como a média dos 10 volumes medidos. O volume total de água armazenado no interior de bromélias de cada espécie por zona de restinga foi calculado como o volume médio armazenado pela espécie multiplicado pela abundância estimada de indivíduos daquela espécie na respectiva zona. Após a retirada da água, pesaram-se as dez bromélias de cada espécie com balanças Pesola com valor máximo de 30, $500 \mathrm{~g} \mathrm{e} 2 \mathrm{~kg}$ (com precisão de 1,5 e $50 \mathrm{~g}$, respectivamente) e com uma balança de valor máximo de $10 \mathrm{~kg}$ (precisão de $100 \mathrm{~g}$ ). A biomassa por zona de restinga foi estimada pela multiplicação da massa média estimada para cada espécie de bromélia pela abundância estimada de indivíduos da espécie presente em cada zona. Diferenças no volume de água e na biomassa entre as espécies foram testadas através de ANOVA, seguida do teste de Tukey para comparações múltiplas (Zar 1999).

\section{Resultados}

$\mathrm{Na}$ área total amostrada da restinga de Jurubatiba, encontraram-se dez espécies de bromélias, pertencentes a cinco gêneros (tabela 1). As espécies de bromélias com maior abundância na restinga de Jurubatiba foram Aechmea nudicaulis, Neoregelia cruenta e Tillandsia stricta (tabela 1). As dez espécies de bromélias encontradas na área amostrada do PNRJ utilizam os mais diversos tipos de substratos; algumas espécies têm hábito epifítico, outras ocupam o solo arenoso das áreas abertas ou o solo coberto por folhiço da área de mata, havendo aquelas que ocupam mais de um tipo de substrato (tabela 1).

Com relação à biomassa das bromélias amostradas, houve diferença significativa entre os valores médios das espécies na área amostrada do PNRJ (ANOVA; $\mathrm{F}=18,445 ; \mathrm{p}<0,001$ ), sendo Bromelia antiacantha a espécie com maior biomassa (tabela 2). Quando comparadas par a par, os valores de biomassa foram significativamente diferentes para algumas espécies (tabela 2). O volume de água no interior das espécies de bromélia na restinga foram significativamente diferentes (ANOVA, $\mathrm{F}=13,534$, $\mathrm{p}<0,001)$. No teste de comparações múltiplas, algumas espécies diferiram significativamente (tabela 2). A espécie de bromélia que teve o maior volume de água reservado no interior do vaso foi Neoregelia cruenta (tabela 2). 
Tabela 1. Valores de abundância, abundância relativa, frequiência de ocorrência e hábito das espécies de bromélias encontradas no Parque Nacional da Restinga de Jurubatiba, Macaé, RJ.

\begin{tabular}{lcccc}
\hline \multicolumn{1}{c}{ Espécies } & $\begin{array}{c}\text { Abundância } \\
\text { absoluta }\end{array}$ & $\begin{array}{c}\text { Abundância } \\
\text { relativa }\end{array}$ & $\begin{array}{c}\text { Freqüência de } \\
\text { ocorrência }\end{array}$ & Hábito \\
\hline Aechmea bromeliifolia (Rudge) Baker & 657 & 0,064 & 0,12 & terrestre \\
Aechmea lingulata (L.) Baker & 1122 & 0,110 & 0,37 & terrestre \\
Aechmea nudicaulis (L.) Grisebach & 2814 & 0,276 & 0,65 & terrestre \\
Bromelia antiacantha Bertoloni & 911 & 0,089 & 0,31 & terrestre \\
Neoregelia cruenta (R. Graham) L.B. Smith & 2388 & 0,235 & 0,73 & terrestre \\
Vriesea neoglutinosa Mez & 512 & 0,050 & 0,23 & terrestre/epífito \\
Tillandsia gardneri Lindley & 138 & 0,013 & 0,25 & epífito \\
Tillandsia recurvata (L.) L. & 50 & 0,005 & 0,03 & epífito \\
Tillandsia stricta Soland in Sims & 1587 & 0,158 & 0,66 & epífito \\
Tillandsia usneoides (L.) L. & $-*$ & $-*$ & 0,02 & epífito \\
Total & 10179 & & & \\
\hline
\end{tabular}

*Não calculadas devido à dificuldade de contar os indivíduos desta espécie.

Tabela 2. Valores médios ( \pm erro padrão da estimativa - ep) de biomassa $(\mathrm{g})$ e volume de água armazenada $(\mathrm{mL})$ nas espécies de bromélias amostradas no Parque Nacional da Restinga de Jurubatiba, Macaé RJ. ( $\mathrm{N}=10)$. Valores com letras sobrescritas diferentes, em cada coluna, indicam haver diferença significativa entre as espécies (comparações múltiplas de Tukey; $\mathrm{p}<0,05$ ).

\begin{tabular}{lcc}
\hline Espécies & $\begin{array}{c}\text { Biomassa } \\
\text { Média } \pm \mathrm{ep}\end{array}$ & $\begin{array}{c}\text { Volume de água } \\
\text { Média } \pm \mathrm{ep}\end{array}$ \\
\hline Aechmea bromeliifolia & $615 \pm 301,89^{\mathrm{a}, \mathrm{b}}$ & $97 \pm 52,66^{\mathrm{a}}$ \\
Aechmea lingulata & $810 \pm 508,15^{\mathrm{a}}$ & $294 \pm 239,73^{\mathrm{b}, \mathrm{c}}$ \\
Aechmea nudicaulis & $280 \pm 181,35^{\mathrm{b}, \mathrm{c}}$ & $178 \pm 127,08^{\mathrm{a}, \mathrm{b}, \mathrm{d}}$ \\
Bromelia antiacantha & $1465 \pm 671,25$ & ${ }_{*}^{* *}$ \\
Neoregelia cruenta & $745 \pm 462,75^{\mathrm{a}, \mathrm{b}}$ & $432 \pm 290,47^{\mathrm{c}}$ \\
Vriesea neoglutinosa & $615 \pm 248,38^{\mathrm{a}, \mathrm{b}}$ & $48 \pm 52,71^{\mathrm{a}, \mathrm{d}}$ \\
Tillandsia gardneri & $56,8 \pm 27,68^{\mathrm{c}}$ & $-*^{* *}$ \\
Tillandsia recurvata & $-{ }^{*}$ & $-{ }^{* *}$ \\
Tillandsia stricta & $28 \pm 14,15^{\mathrm{c}}$ & $-{ }^{* *}$ \\
Tillandsia usneoides & $-{ }^{*}$ & $-*^{* *}$ \\
\hline
\end{tabular}

* Massa dos indivíduos não determinada no campo.

** Não se aplica.

A composição das espécies de bromélia variou entre as zonas da restinga, sendo as zonas AAE e MPI (ambas possuindo nove espécies de bromélias) as áreas com maior riqueza de espécies, seguidas da zona AAC, com riqueza de oito espécies. A zona
PHR foi a única das zonas de vegetação da restinga de Jurubatiba onde não ocorreu nenhuma espécie de bromélia.

$\mathrm{Na}$ área total amostrada na restinga, ocorreu um total de 10179 indivíduos de bromélias, tendo a abundância de cada espécie diferido entre as zonas de vegetação (figura 3 ). A densidade total de bromélias diferiu estatisticamente $\left(\chi^{2}=4422,9 ; p<0,001\right)$ entre as zonas. As zonas de vegetação com maior densidade foram a AAE, com 15725 ind.ha $^{-1}$ $(\mathrm{N}=2516)$, seguida da zona AAC com 11900 ind.ha ${ }^{-1}(\mathrm{~N}=5236)$.

A zona de vegetação $\mathrm{AAE}$ foi a área com a maior diversidade $\left(\mathrm{H}^{\prime}=2,358\right)$ e equitabilidade $(0,786)$, enquanto a zona FPP teve o menor índice de diversidade $\left(\mathrm{H}^{\prime}=1,484\right)$ e equitabilidade $(0,639)$ (tabela 3). A maior biomassa de bromélias por zona de vegetação foi encontrada na zona FPP (tabela 3), onde as espécies mais abundantes, Bromelia antiacantha e Neoregelia cruenta, possuíram os maiores valores de biomassa. A zona AAC teve o menor valor de biomassa total (tabela 3 ).

A zona de vegetação em que houve maior volume estimado de água reservado no interior de bromélias foi a AAE e a com a menor quantidade de água estimada no interior de bromélias foi a zona FPP (tabela 3).

Os valores de similaridade entre as zonas variaram de 0,55 a 0,89, sendo as zonas AAC e AAE as 
mais similares na composição de espécies de bromélias (tabela 4).

\section{Discussão}

A variação espacial indicada pelo crescente aumento na riqueza de espécies de bromeliáceas desde a zona PHR até o cordão de mata (MPI) pode estar relacionada, em parte, com a diminuição da salinidade e da deposição de salsugem, que ocorre em
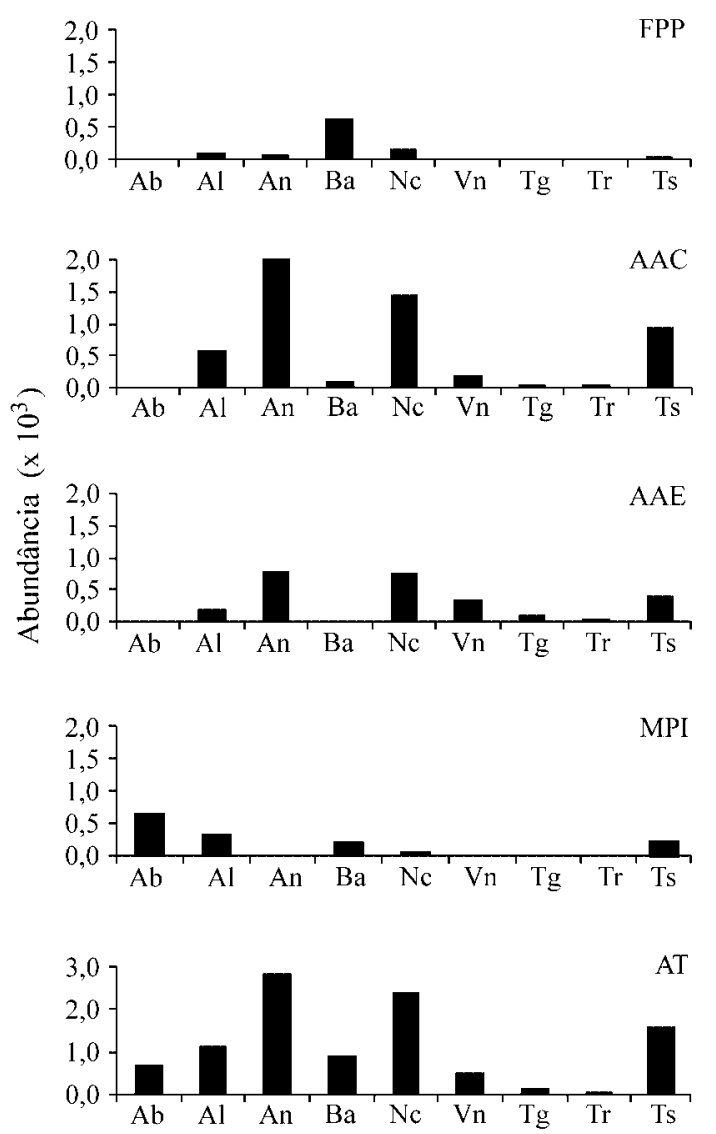

Figura 3. Distribuição de freqüência das espécies de bromélias por zona de vegetação de restinga da área amostrada do Parque Nacional da Restinga de Jurubatiba. $\mathrm{Ab}=$ Aechmea bromeliifolia; $\mathrm{Al}=$ A. lingulata $; \mathrm{An}=$ A. nudicaulis $; \mathrm{Ba}=$ Bromelia antiacantha; $\mathrm{Nc}=$ Neoregelia cruenta $; \mathrm{Vn}=$ Vriesea neoglutinosa; $\mathrm{Tg}=$ Tillandsia gardneri $; \mathrm{Tr}=T$. recurvata $; \mathrm{Ts}=T$. stricta $; \mathrm{FPP}$ = área de vegetação fechada do pós-praia; AAC = área aberta de Clusia; $\mathrm{AAE}=$ área aberta de Ericácea; $\mathrm{MPI}=$ mata periodicamente inundada; AT $=$ abundância total. A espécie $T$. usneoides não foi incluída devido à dificuldade de contar os indivíduos. grande quantidade na zona PHR e decresce em direção às zonas interiores (Hay \& Lacerda 1984, Pammenter 1984, Gómez \& Winkler 1991), e com a variação espacial na disponibilidade de suporte para as espécies de bromélias epífitas. Os dados no presente estudo indicam que, para a restinga de Jurubatiba, as bromélias não constituem espécies da zona halófila psamófila. Outros estudos também têm mostrado que espécies desta família não são observadas nesta zona de restinga (Rawitscher 1944, Pfadenhauer 1978, Waechter 1985, Henriques et al. 1986, Araujo \& Oliveira 1988, Araujo et al. 1998), a qual está susceptível à ação das ondas em épocas de tempestades (Araujo 1992) e à alta salinidade (Pammenter 1984), sendo o solo bastante pobre em nutrientes e água (Waechter 1985).

A presença de moitas acarreta um aumento de sombreamento e de porcentagem de cobertura vegetal, oferecendo microhabitats com maior disponibilidade de nutrientes, umidade e temperatura mais amena (Franco et al. 1984, Hay \& Lacerda 1984, Fialho \& Furtado 1993, Montezuma 1997, Zaluar 1997) e substratos potenciais para as espécies epífitas. A maior riqueza de bromélias nas zonas de vegetação AAC, AAE e MPI pode ser explicada pela maior cobertura do solo pelas moitas (Henriques et al. 1986, Montezuma 1997, Araujo et al. 1998), altura do dossel e presença de substratos para epífitas, que cria diferentes e particulares condições para o estabelecimento de maior número de espécies de bromélias. No entanto, embora a zona FPP também possua um grande percentual de cobertura vegetal, com uma vegetação mais fechada e impenetrável (Henriques et al. 1986, Araujo et al. 1998), esta é mais susceptível aos efeitos do salsugem devido à maior proximidade da linha de praia, possuindo, então, menor riqueza e abundância de bromélias.

A espécie heliófila $T$. stricta, que suporta alta incidência de luz direta (Leme 1984), provavelmente ocorre no interior das moitas desta restinga por ser sensível aos efeitos intensos da salinidade e por encontrar nesses microhabitats substratos apropriados para se estabelecer. Bromelia antiacantha ocorreu basicamente nas zonas FPP e MPI, assim como no estudo de Araújo et al. (1998). A presença de indivíduos desta espécie na zona FPP sugere maior tolerância à alta salinidade. As espécies N. cruenta e A. nudicaulis ocorrem em elevada abundância na 
Tabela 3. Sumário dos valores de abundância, riqueza, densidade (ind.ha $\left.{ }^{-1}\right)$, diversidade $\left(\mathrm{H}^{\prime}\right)$, equitabilidade, biomassa $\left(\mathrm{kg}^{-h^{-1}} \mathrm{e}^{-1} \mathrm{e}\right.$ volume de água armazenada (litros.ha ${ }^{-1}$ ) de Bromeliaceae em cada zona de vegetação de restinga estudada no Parque Nacional da Restinga de Jurubatiba, Macaé, RJ. O valor entre parênteses indica a área amostrada em cada zona: FPP = fechada de pós-praia; $\mathrm{AAC}=$ área aberta de Clusia $; \mathrm{AAE}=$ área aberta de Ericácea; $\mathrm{MPI}$ = mata periodicamente inundada.

\begin{tabular}{lcccc}
\hline \multicolumn{1}{c}{ Parâmetros } & FPP $(0,14$ ha $)$ & AAC $(0,44$ ha $)$ & AAE $(0,16$ ha $)$ & MPI $(0,17$ ha $)$ \\
\hline Abundância & 907 & 5236 & $2516^{*}$ & 1520 \\
Riqueza & 5 & 8 & 9 & 9 \\
Densidade total & 6478 & 11900 & $15725^{*}$ & $8941^{*}$ \\
Diversidade de Shannon (H') & 1,484 & 2,174 & 2,358 & 2,102 \\
Equitabilidade (J) & 0,639 & 0,725 & 0,786 & 0,701 \\
Biomassa total & 7721,2 & $5337,6^{* *}$ & $7129,6^{* *}$ & $6080,7^{* *}$ \\
Volume de água & 671,8 & 2597,7 & 3294,8 & 1105,4 \\
\hline
\end{tabular}

* T. usneoides não incluída devido à dificuldade de contagem dos indivíduos.

** T. usneoides e T. recurvata não incluídas devido à dificuldade de medir a massa dos indivíduos no campo.

região entre-moitas das zonas AAC e AAE no PNRJ, o que também pode ser observado por Henriques $e t$ al. (1986). Tal fato sugere que estas duas espécies possuem capacidade de sobreviver a altas incidências de luminosidade e elevada temperatura da areia, pois acumulam uma grande quantidade de água entre as suas folhas, o que manteria a temperatura mais amena, além de facilitar a absorção de nutrientes através de tricomas presentes nas folhas (Benzing 1980). Alguns estudos (Hay \& Lacerda 1980, 1984, Zaluar 1997) mostram que algumas espécies de bromélias em restinga são capazes de se estabelecer nos primeiros estágios de sucessão do desenvolvimento de moitas, pois absorvem os nutrientes através de células especializadas nas folhas (Benzing 1980, Hay \& Lacerda 1984).

Das dez espécies de bromélias ocorrentes no PNRJ amostradas neste estudo, quatro (T. stricta, $T$. usneoides, $T$. gardneri e $V$. neoglutinosa) possuem larga distribuição no litoral brasileiro, ocorrendo nas porções sul e norte do litoral (Aragão 1967). As espécies $B$. antiacantha e A. nudicaulis ocorrem restritas à porção sul do litoral do Brasil (de Ilhéus para o sul) (Aragão 1967), sendo a primeira presente em todo o litoral do Rio de Janeiro (Araujo \& Henriques 1984). No estado do Rio de Janeiro, a espécie A. lingulata ocorre nas restingas de Cabo Frio (Fontoura et al. 1991), Macaé, Araruama (Araujo \& Henriques 1984) e Massambaba (L. Cogliatti-Carvalho et al., dados não publicados). Aechmea nudicaulis e $N$. cruenta ocorrem nas restingas de Macaé, Cabo Frio, Maricá (Araujo \& Henriques 1984, Fontoura et al. 1991) e apenas a segunda espécie em Grumari (Fontoura et al. 1991). No estado do Rio de Janeiro, A. bromeliifolia tem sua distribuição nas restingas de São João da Barra e Macaé (Fontoura et al. 1991). Tillandsia recurvata ocorre, no estado do Rio de Janeiro, em Macaé, Cabo Frio, Araruama e Grumari (Araujo \& Henriques 1984).

As zonas AAC e AAE foram as mais similares em termos da composição de espécies de bromélias, o que seria esperado já que ambas são contínuas e estruturalmente similares, sendo caracterizadas pela formação de moitas esparsas. Localidades próximas

Tabela 4. Valores do índice de similaridade de Jaccard entre as quatro zonas de vegetação de restinga estudadas no Parque Nacional da Restinga de Jurubatiba, Macaé, RJ. FPP = fechada de pós-praia; AAC = área aberta de Clusia; AAE = área aberta de Ericácea; MPI = mata periodicamente inundada.

\begin{tabular}{cccc}
\hline Zonas & AAC & AAE & MPI \\
\hline FPP & 0,62 & 0,56 & 0,55 \\
AAC & & 0,89 & 0,70 \\
AAE & & & 0,80 \\
\hline
\end{tabular}


geralmente refletem maior similaridade florística (Pereira \& Araujo 1995). Embora na zona AAC tenha ocorrido a maior abundância total de espécies, a zona AAE teve a maior densidade total. A AAE sofre menos influência do mar e está mais protegida da ação do vento quando comparado à AAC, o que pode amenizar a ação deletéria destes fatores ao desenvolvimento da vegetação (Montezuma 1997). Além disso, a presença do lençol freático pouco profundo na zona AAE propicia a formação de moitas (Henriques et al. 1986, Pereira \& Araujo 1995, Montezuma 1997), criando microhabitats com condições de luminosidade, temperatura, umidade e salinidade mais favoráveis para o estabelecimento das espécies de bromélias. Em áreas mais abertas como a AAC, o espaçamento entre as manchas de vegetação é maior (Zaluar 1997), elevando os níveis de incidência luminosa e de temperatura, o que pode representar condições adversas para a sobrevivência de diversas espécies vegetais (Fialho 1990). Além disso, a AAC possui um menor percentual de matéria orgânica e nutrientes no solo do que a AAE, embora estes sejam, de modo geral, baixos nestas duas zonas (Hay \& Lacerda 1984). A abundância, a diversidade e a densidade de bromélias são menores na MPI do que em AAC e AAE, provavelmente devido à maior discrepância entre as abundâncias de bromélias terrestres e epífitas naquela zona. Na zona de MPI, a dominância é de espécies terrestres, com maior representatividade de apenas duas espécies ( $A$. lingulata e $A$. bromeliifolia), enquanto as densidades das espécies epífitas são bastante baixas. A MPI possui maior quantidade de nutrientes e água no solo, sendo uma zona supostamente mais úmida quando comparada à região de moitas e entre moitas nas zonas AAC e AAE (Henriques et al. 1986). Algumas espécies ( $V$. neoglutinosa) são inexistentes na MPI, enquanto que nas demais zonas (AAC e $\mathrm{AAE}$ ) a presença de epífitas promove maior verticalização na utilização do ambiente pelas plantas. As áreas com menor índice de similaridade de bromélias (FPP e MPI) foram as mais distantes entre si, e são compostas por tipos de vegetação, condições edáficas e de salinidade bastante peculiares a cada zona (Araujo et al. 1998).

A maior biomassa de bromélias encontrada na zona FPP ocorreu provavelmente devido à elevada densidade nesta zona da espécie $B$. antiacantha, que possui o maior valor de biomassa entre as espécies de bromélias amostradas. AAE foi a zona de vegetação que possuiu o maior volume de água armazenado por hectare, e este fato provavelmente se deve à presença das espécies de bromélia-tanque $N$. cruenta, A. nudicaulis e A. lingulata, que ocorreram em alta densidade nesta zona e possuem elevada capacidade de reserva de água. Diversos estudos têm indicado que as bromélias-tanque são uma importante fonte de recursos para várias espécies que vivem diretamente associadas a elas (Picado 1913, Lopez 1997, Oliveira \& Rocha 1997, Richardson 1999) ou que passarão parte do dia ou da noite no interior do vaso (Britto-Pereira et al. 1988), especialmente em locais onde o ambiente externo é pouco favorável ao desenvolvimento e sobrevivência destes organismos (Fialho 1990, Fialho \& Furtado 1993, Oliveira et al. 1994 a, b, Oliveira \& Rocha 1997). O volume de água armazenado no vaso de bromélias depende da forma da roseta, da disposição das folhas e do tamanho e grau de dilatação das bainhas foliares (Leme 1984), características que, no caso de $N$. cruenta, A. nudicaulis e A. lingulata, permitem o acúmulo de um grande volume de água. Além disso, na AAE, o nível do lençol freático é mais alto, chegando a tornar o terreno alagado em épocas de chuva (Henriques et al. 1986, Montezuma 1997), o que pode provavelmente contribuir para o maior volume de água no vaso das bromélias-tanque nesta zona, já que torna o ambiente menos xérico. Em ambientes como as restingas, em que as condições ambientais são extremas devido à alta temperatura e à alta exposição à luz solar (Fialho 1990), as espécies de bromélias que armazenam grandes volumes de água, como N. cruenta, A. nudicaulis e A. lingulata, tornam-se uma importante fonte deste recurso para diversas outras espécies animais e vegetais.

Conclui-se que a estrutura e a composição de Bromeliaceae do Parque Nacional da Restinga de Jurubatiba varia fortemente entre as cinco zonas estudadas, devido ao aumento da complexidade do ambiente com o distanciamento da linha de praia, o que favorece a ocorrência de maior número de espécies, especialmente de bromélias epífitas. Os dados também sugerem que cada zona é composta por um conjunto particular de espécies de bromélias, resultando na caracterização de cada uma das zonas de vegetação com base na ocorrência de espécies de 
Bromeliaceae dominantes em cada zona entre aquelas existentes nesta restinga.

Agradecimentos - Este estudo é parte da tese de Doutorado do primeiro autor no Programa de Pós-Graduação em Ecologia da UFRJ, dentro do Projeto Ecologia de Restingas Brasileiras, do Programa de Ecologia, Conservação e Manejo de Ecossistemas do Sudeste Brasileiro e do Projeto Vertebrados do Leste Brasileiro, Setor de Ecologia, Instituto de Biologia Roberto Alcântara Gomes, da Universidade do Estado do Rio de Janeiro e foi parcialmente subvencionado com Auxílio à Pesquisa da Fundação de Amparo à Pesquisa do Estado do Rio de Janeiro (FAPERJ), processo $\mathrm{n}^{\mathrm{o}}$ E-26/171.885/1999 e do CNPq (46197/00-7). E. Leme e A. Costa identificaram as espécies de Bromeliáceas. Durante o estudo, A.F.N. Freitas (Registro CAPES nํ990207-7) e L. Cogliatti-Carvalho (Processo CNPq n⿳‥ 140727/1999-0) receberam bolsas de mestrado. C.F.D. Rocha (Processo $\mathrm{n}^{\mathrm{0}} 300$ 819/94-3) e M. Van Sluys (Processo n⿳⺈ 301117/95-0) receberam bolsas de produtividade em pesquisa do Conselho Nacional do Desenvolvimento Científico e Tecnológico - CNPq.

\section{Referências bibliográficas}

ARAGÃO, M.B. 1967. Condições de habitat e distribuição geográfica de algumas Bromeliaceae. Sellowia 19:83-95.

ARAUJO, D.S.D., SCARANO, F.R., SÁ, C.F.C., KURTZ, B.C., ZALUAR, H.L.T., MONTEZUMA, R.C.M. \& OLIVEIRA, R.C. 1998. Comunidades vegetais do Parque Nacional da Restinga de Jurubatiba. In Ecologia das lagoas costeiras do Parque Nacional da Restinga de Jurubatiba e do Município de Macaé (RJ) (F.A. Esteves, ed.). Universidade Federal do Rio de Janeiro, Rio de Janeiro, p.39-62.

ARAUJO, D.S.D. \& HENRIQUES, R.P.B. 1984. Análise florística das restingas do Estado do Rio de Janeiro. In Restingas: origem, estrutura, processos (L.D. Lacerda, D.S.D. Araujo, R. Cerqueira \& B. Turcq, orgs.). CEUFF, Niterói, p.159-193.

ARAUJO, D. \& OLIVEIRA, R. 1988. Reserva Biológica Estadual da Praia do Sul (Ilha Grande, Estado do Rio de Janeiro): lista preliminar da flora. Acta Botânica Brasilica $1: 83-94$.

ARAUJO, D.S.D. 1992. Vegetation types of sandy coastal plains of tropical Brazil: a first approximation. In Coastal plant communities of Latin America (U. Seelinger, ed.). Academic Press, San Diego, p.337-347.

BENZING, D.H. 1980. The Biology of bromeliads. Mad River Press, California.

BRITTO-PEREIRA, M.C., CERQUEIRA, R., SILVA, H.R. \& CARAMASCHI, U. 1988. Anfíbios anuros da restinga de Barra de Maricá, RJ: levantamento e observações preliminares sobre a atividade reprodutiva das espécies registradas. In Anais do V seminário regional de ecologia. Universidade de São Carlos, São Carlos, p.295-306.

DANSEREAU, P. 1947. Zonation et succession sur la restinga de Rio de Janeiro. - I. Halosère. Revue Canadienne de Biologie 6:448-477.

FIALHO, R.F. 1990. Seed dispersal by a lizard and a treefrog Effect of dispersal site on seed survivorship. Biotropica $22: 423-424$
FIALHO, R.F \& FURTADO, A.L.S. 1993. Germination of Erythroxylum ovalifolium (Erythroxylaceae) seeds within the terrestrial bromeliad Neoregelia cruenta. Biotropica 25:359-362.

FONTOURA, T., COSTA, A. \& WENDT, T. 1991. Preliminary checklist of the Bromeliaceae of Rio de Janeiro State, Brazil. Selbyana 12:5-45.

FRANCO, A.C., VALERIANO, D.M., SANTOS, F.M., HAY, J.D., HENRIQUES, R.P.B. \& MEDEIROS, R.A. 1984. Os microclimas das zonas de vegetação da praia da restinga de Barra de Maricá, Rio de Janeiro. In Restingas: Origem, estrutura e processos. (L.D. Lacerda, D.S.D. Araujo, R. Cerqueira \& B. Turcq, orgs.). CEUFF, Niterói, p.413-425.

GÓMEZ, M.A. \& WINKLER, S. 1991. Bromelias en manglares del Pacífico de Guatemala. Revista de Biologia Tropical 39:207-214

HAY, J.D. \& LACERDA, L.D. 1980. Alterações nas características do solo após a fixação de Neoregelia cruenta (R. Gran.) L. Smith (Bromeliaceae) em um ecossistema de restinga. Ciência e Cultura 32:863-867.

HAY, J.D. \& LACERDA, L.D. 1984. Ciclagem de nutrientes no ecossistema de restinga. In Restingas: Origem, estrutura e processos (L.D. Lacerda, D.S.D. Araujo, R. Cerqueira \& B. Turcq, orgs.). CEUFF, Niterói, p.459-473.

HENRIQUES, R.P.B., MEIRELLES, M.L. \& HAY, J.D. 1984. Ordenação e distribuição de espécies das comunidades vegetais na praia da restinga de Barra de Maricá, Rio de Janeiro. Revista Brasileira de Botânica. 7:27-36.

HENRIQUES, R.P.B., ARAUJO, D.S.D. \& HAY, J.D. 1986. Descrição e classificação dos tipos de vegetação da restinga de Carapebus, Rio de Janeiro. Revista Brasileira de Botânica. 9:173-189.

LACERDA, L.D., ARAUJO, D.S.D. \& MACIEL, N.C. 1993. Dry coastal ecosystems of the tropical Brazilian coast. In Dry coastal-ecosystems: Africa, Asia, Oceania (E. Van der Maarel, ed.). Elsevier, Amsterdam, p.477-493.

LEME, E.M.C. 1984. Bromélias. Ciência Hoje 3:66-72.

LOPEZ, L.C.S. 1997. Comunidades aquáticas em tanques de bromélias: zonação e sucessão. Dissertação de mestrado, Universidade Federal do Rio de Janeiro, Rio de Janeiro.

MAGURRAN, A.E. 1988. Ecological diversity and its measurement. Cambridge University Press, Cambridge.

MARTINS, L., SUGUIO, K. \& FLEXOR, J.M. 1993. As flutuações de nível do mar durante o quaternário superior e a evolução geológica de "deltas" brasileiros. Boletim do Instituto de Geologia - USP, Publicações Especiais 15:1-186.

MONTEZUMA, R.C.M. 1997. Estrutura da vegetação de uma restinga de Ericaceae no município de Carapebus, RJ. Dissertação de mestrado, Universidade Federal do Rio de Janeiro, Rio de Janeiro.

OLIVEIRA, M.G.N., ROCHA, C.F.D. \& BAGNALL, T. 1994a Bromélias-tanque servem de abrigo para espécies. Ciência Hoje 17:21-22.

OLIVEIRA, M.G.N., ROCHA, C.F.D. \& BAGNALL, T. 1994b. A comunidade animal associada à bromélia-tanque Neoregelia cruenta (R. Graham) L.B. Smith. Bromélia 1:22-29. 
OLIVEIRA, M.G.N. \& ROCHA, C.F.D. 1997. O efeito da complexidade da bromélia-tanque Neoregelia cruenta (R. Graham) L.B. Smith sobre a comunidade animal associada. Bromélia 4:13-22.

ORMOND, W.T. 1960. Ecologia das restingas do Sudeste do Brasil: comunidades vegetais das praias arenosas. Parte I. Arquivos do Museu Nacional 50:185-236.

PAMMENTER, N.W. 1984. Ecological and physiological aspects of plant communities of the sand dunes of the east coast of Sothern Africa. In Restingas: Origem, estrutura e processos (L.D. Lacerda, D.S.D. Araujo, R. Cerqueira \& B. Turcq, orgs.). CEUFF, Niterói, p.425-440.

PEREIRA, O.J. \& ARAUJO, D.S.D. 1995. Estrutura da vegetação de entre moitas da formação aberta de Ericaceae no Parque Estadual de Setiba, ES. Oecologia Brasiliensis. Estrutura, Funcionamento e Manejo de Ecossistemas Brasileiros 1:245-257

PETRUCIO, M.M. \& FARIA, M.F. 1998. Concentrações de carbono orgânico, nitrogênio total e fósforo disponível no sedimento das Lagoas Cabiúnas e Comprida. Comunidades Vegetais do Parque Nacional da Restinga de Jurubatiba. In Ecologia das Lagoas Costeiras do Parque Nacional da Restinga de Jurubatiba e do Município de Macaé (RJ) (F.A. Esteves, ed.). Universidade Federal do Rio de Janeiro, Rio de Janeiro, p.135-144.

PFADENHAUER, J. 1978. Contribuição ao conhecimento da vegetação e de suas condições de crescimento nas dunas costeiras do Rio Grande do Sul, Brasil. Revista Brasileira de Biologia 38:827-836.
PICADO, C. 1913. Les broméliacées épiphytes considérées comme milieu biologique. Bulletin Scientifique France et Belgique 5:215-360.

RAWITSCHER, F.K. 1944. Algumas noções sobre a vegetação do litoral brasileiro. Boletim da Associação dos Geógrafos Brasileiros 5:13-28.

RICHARDSON, B.A. 1999. The bromeliad microcosm and the assessment of faunal diversity in a Neotropical Forest. Biotropica 31:321-336.

RIVERO, J.A. 1989. Two beautiful bromeliad frogs from Andes of Venezuela. Journal of Bromeliad Society 39:26-27.

ROCHA, C.F.D., COGLIATTI-CARVALHO, L., ALMEIDA, D.R. \& FREITAS, A.F.N. 1997. Bromélias: ampliadoras da biodiversidade. Bromélia 4:7-10.

SILVA, J.G. \& SOMNER, G.V. 1984. A vegetação de restinga na Barra de Maricá, RJ. In Restingas: origem, estrutura, processos (L.D. Lacerda, D.S.D. Araujo, R. Cerqueira \& B. Turcq, orgs.). CEUFF, Niterói, p.217-225.

SMART, J. 1938. Note on the insect fauna of the bromeliad Brocchinia micrantha (Baker) Mez of British Guyana. Entomological Monographies 74:198-200.

WAECHTER, J.L. 1985. Aspectos ecológicos da vegetação de restinga no Rio Grande do Sul, Brasil. Comunidade do Museu Científico. PUCRS, Série Botânica, Porto Alegre 33:49-68.

ZALUAR, H.L.T. 1997. Espécies focais e a formação de moitas na restinga aberta de Clusia, Carapebus, RJ. Dissertação de mestrado, Universidade Federal do Rio de Janeiro, Rio de Janeiro.

ZAR, J.H. 1999. Biostatistical analysis. Prentice Hall, New Jersey. 